\title{
ORIGINAL
}

\section{UTILIZACIÓN DE LOS INHIBIDORES DE LA ACETILCOLINESTERASA Y LA MEMANTINA PARA EL TRATAMIENTO CLÍNICO DE LA DEMENCIA TIPO ALZHEIMER}

Manuel Portela Romero (1), Julio Pombo Romero (1), Rosendo Bugarín González (2), Margarita Tasende Souto (1) y Silvia Represa Veiga (1)

(1) Dirección General de Farmacia y Productos Sanitarios de la Consejería de Sanidad de Galicia.

(2) División de Asistencia Sanitaria. Servicio Gallego de Salud.

\section{RESUMEN}

Fundamento: En los últimos años han aumentado las alternativas terapéuticas en el tratamiento de la demencia tipo Alzheimer (DTA), por lo que se hace necesario conocer el patrón de utilización de estos nuevos medicamentos.

Métodos: Estudio observacional transversal de la población con DTA tratada con inhibidores de la acetilcolinesterasa y/o memantina en el Servicio Gallego de Salud. Se construyeron bases de datos con las variables demográficas y datos de utilización durante abril de 2005 de los referidos medicamentos.

Resultados: De 5.110 pacientes con DTA, el 70,47\% eran mujeres. La edad media de éstas fue de 80,12 y de 78,61 en los hombres, con desviaciones típicas de 6,66 y 7,03 respectivamente. El sexo femenino se mostró como un factor asociado a presentar DTA a trafemenino se mostró como un factor asociado a presentar DTA a tra-
tamiento farmacológico (OR: 1,932 [IC $95 \%: 1,819-2,052]$ ). El medicamento más utilizado fue donepezilo $(44,46 \%$ de los pacientes). Se hallaron diferencias significativas en el porcentaje de pacientes con memantina a favor de los pacientes $\leq 64$ años $(19,7 \%$ en $\leq 64$ años vs. $14 \%$ en $>64$ años $[\mathrm{p}<0,05])$, y de donepezilo a favor de los pacientes $>95$ años $(75 \%$ en 395 años vs. $46,9 \%$ en $>95$ años $[\mathrm{p}<0,03])$. No se observaron diferencias significativas en la utilización de la terapéutica antidemencia, expresada en DDD/paciente/día entre sexos, grupos de edad, ni en la desagregación de los grupos de edad por sexos.

Conclusiones: La utilización de memantina (en monoterapia o en combinación) es más frecuente en menores de 64 años. El sexo femenino se mostró como un factor asociado a presentar DTA a tratamiento farmacológico.

Palabras clave: Enfermedad de Alzheimer. Galantamina. Memantina. Inhibidores de la Colinesterasa.

Correspondencia:

Manuel Portela Romero

Centro de Información Farmacoterapéutica

Consellería de Sanidade

Edificio Administrativo San Lázaro

Santiago de Compostela

15703 A Coruña

Correo electrónico: manuel.portela.romero@sergas.es
ABSTRACT

\section{Use of Acetylcholinesterase Inhibitors and Memantine in Alzheimer-type Dementia}

Background: The therapeutic options to treat Alzheimer-type dementia (ATD) in recent years, have made it increasingly necessary to become familiar with the employment pattern of these new medicinal products.

Methods: A cross-sectional observational study of the population with ATD treated with acetylcholinesterase inhibitors and/or memantine in the Gallician Health Service. Databases were compiled with the demographic variables and use data during April 2005 of the medicinal products studied.

Results: Of the 5,110 patients with ATD, 70.47\% were women. The mean age of the women was 80.12 and of the men was 78.61 years old, with standard deviations of 6.66 and 7.03 , respectively. The female sex was one of the factors associated with presenting ATD to pharmacological treatment (OR: 1.932 [CI 95\%:1.819$2.052])$. The medication used most was donepezyl $(44.46 \%$ of patients). Significant differences were found in that memantine was used to treat more patients in the group of patients $\leq 64$ years $(19.7 \%$ in $\leq 64$ years vs. $14 \%$ in $>64$ years $[p<0.05]$ ), and donepezyl was preferentially used in patients $\geq 95$ years $(75 \%$ in $\geq 95$ years vs. $46.9 \%$ ferentially used in patients $\geq 95$ years $(75 \%$ in $\geq 95$ years vs. $46.9 \%$
in $<95$ years $[p<0,03])$. No significant differences were observed in the use of anti-dementia therapy, expressed in DDD/patient/day between the sexes, age groups, or in the age groups separated according to sex.

Conclusions: The use of memantine (in monotherapy or in combination) is more frequent in patients under 64 years old. Female sex was found to be a factor associated with presenting ATD to pharmacological treatment.

Key words: Alzheimer disease. Cholinesterase inhibitors. Galantamine. Memantine. 


\section{INTRODUCCIÓN}

La demencia es un síndrome adquirido que se caracteriza por un deterioro subagudo o crónico, progresivo, global e irreversible de la función cerebral, con preservación del estado de conciencia. Habitualmente produce pérdida de memoria, pérdida de las funciones ejecutivas y cambios en la personalidad ${ }^{1}$.

La demencia tipo Alzheimer (DTA) es la más frecuente en la población mayor de 64 años en los países occidentales, representando el $50-70 \%$ de los casos $^{2}$ diagnosticados, seguida por la demencia vascular y la demencia mixta ${ }^{3-5}$.

El manejo terapéutico de la DTA se centra en el tratamiento sintomático del deterioro cognitivo o de los problemas de comportamiento afectivo. En la actualidad, los inhibidores de la acetilcolinesterasa están indicados para el tratamiento de la DTA de intensidad leve a moderada ${ }^{6,7}$. Este grupo terapéutico incluye tacrina (autorizada el año 1993), aunque actualmente de baja por las reacciones de hepatotoxicidad asociadas a su uso ${ }^{8}$, donepezilo (año 1998), rivastigmina (año 2000), galantamina (año 2001). En el año 2003 se autorizó la memantina, un antagonista no competitivo del receptor N-metil-D-aspartato (NMDA), siendo en la actualidad el único medicamento antidemencia con la indicación de DTA de intensidad moderada a grave ${ }^{9}$.

El propósito de nuestro trabajo es conocer la prevalencia de los pacientes con DTA a tratamiento con inhibidores de la acetilcolinesterasa y/o memantina y el patrón de utilización de estos fármacos en la práctica clínica en nuestro medio. Por otra parte, pretendemos cuantificar la relación de las variables sexo y edad con la prevalencia de la DTA y los patrones de utilización terapéutica en esta enfermedad.

\section{SUJETOS Y MÉTODO}

Estudio observacional transversal donde se analizan las características demográficas y datos de utilización de las distintas opciones farmacoterapéuticas en el tratamiento de los pacientes con diagnóstico de DTA en Galicia. En las opciones farmacoterapéuticas se incluyen los grupos terapéuticos ATC N06DA (medicamentos antidemencia, inhibidores de la acetilcolinesterasa: donepezilo, galantamina, rivastigmina) y N06DX (otros medicamentos antidemencia: memantina).

El ámbito de estudio es la Comunidad Autónoma de Galicia, con una población de 2.695.880, según el censo del año $2001^{10}$.

La población a estudio es toda la población con derecho a prestación farmacéutica con cargo al Servicio Gallego de Salud (2.659.688 personas en el año 2004) ${ }^{11}$. Dentro de ésta, todos los pacientes con diagnóstico clínico de DTA que están siendo tratados con medicamentos antidemencia de los grupos terapéuticos ATC N06DA y N06D han sido incluidos en el registro informatizado de homologación de prescripciones en receta médica (visado), centralizado en la Dirección General de Farmacia y Productos Sanitarios de la Consellería de Sanidade de la Xunta de Galicia.

Para la inclusión de pacientes en este registro el diagnóstico clínico debe ser realizado por médicos de atención especializada, ya que en la actualidad los medicamentos antidemencia están clasificados como «Diagnóstico Hospitalario».

El periodo de estudio es el mes abril del año 2005.

Las variables recogidas en los pacientes con diagnóstico de DTA fueron: número de casos, edad, sexo, medicamentos antidemencia utilizados (por principio activo), dosis diaria definida consumida por paciente y día (DDD/paciente/día).

La unidad de DDD de cada principio activo se ha obtenido del Collaborating Centre for Drug Statistics Methodology de la $\mathrm{OMS}^{12}$. 
Análisis estadístico: para el procesamiento de los datos se construyeron bases de datos en los programas Bussiness Objects, EXCEL 5.0, EPIDAT 3.0 y SPSS 12.0. Se calcularon los estadísticos descriptivos para la media y la desviación típica y sus correspondientes intervalos de confianza al $95 \%$ para las variables objeto del estudio. Se contrastaron hipótesis de igualdad sobre los parámetros de estudio entre subgrupos de la muestra (grupos de edad y sexo), se realizó la prueba ji-cuadrado para comprobar la influencia de la edad y el sexo en el tipo de tratamiento utilizado, así mismo se calculó el odds ratio, la razón de prevalencias y se efectuó la prueba ji-cuadrado para establecer el riesgo de padecer DTA asociado al sexo.

Declaración de confidencialidad de los datos: se ha respetado en todo momento la confidencialidad de los datos, de manera que se garantiza el anonimato de los pacientes. Para ello, los datos analizados se han extraído del registro informatizado en una única vez utilizando una estrategia de extracción, de acuerdo con los criterios de búsqueda seleccionados, que no incluía variables que pudieran identificar a los pacientes.

\section{RESULTADOS}

El resumen de los resultados del estudio se muestra en la tabla 1. El número de pacientes con diagnóstico de DTA incluidos en el registro informatizado de prescripciones del Servicio Gallego de Salud fue de 5.110 , de los cuales 3.601 eran mujeres $(70,47 \%)$. La edad media en las mujeres fue de 80,12 años, con una desviación típica de 6,66 años, mientras que la edad media en los hombres fue de 78,61 años, con una desviación típica de 7,03 años.

La prevalencia de la DTA a tratamiento farmacológico en la población mayor de 64 años fue $1,03 \%$ en las mujeres y del $0,61 \%$ en los hombres. El estudio de datos de pre-

Tabla 1

Características de las personas estudiadas

\begin{tabular}{|l|c|c|c|}
\hline & Hombres & Mujeres & Total \\
\hline Número de personas con DTA que realizan & $1.509(29,53 \%)$ & $3.601(70,47 \%)$ & 5110 \\
algún tratamiento antidemencia & 78,61 & 80,12 & 79,75 \\
\hline Edad media (años) & $683(45,26 \%)$ & $1589(44,12 \%)$ & $2272(44,46 \%)$ \\
\hline Tratados con Donepezilo & $363(24,05 \%)$ & $876(24,32 \%)$ & $1239(24,24 \%)$ \\
\hline Tratados con Rivastigmina & $266(17,62 \%)$ & $625(17,35 \%)$ & $891(17,43 \%)$ \\
\hline Tratados con Galantamina & $103(6,82 \%)$ & $324(8,99 \%)$ & $427(8,35 \%)$ \\
\hline Tratados con Memantina & $50(3,31 \%)$ & $100(2,77 \%)$ & $150(2,93 \%)$ \\
\hline Tratados Memantina+Donepezilo & $27(1,78 \%)$ & $54(1,49 \%)$ & $81(1,58 \%)$ \\
\hline Tratados Memantina+Rivastigmina & $17(1,12 \%)$ & $33(0,92 \%)$ & $50(0,98 \%)$ \\
\hline Tratados Memantina+Galantamina & & & \\
\hline
\end{tabular}




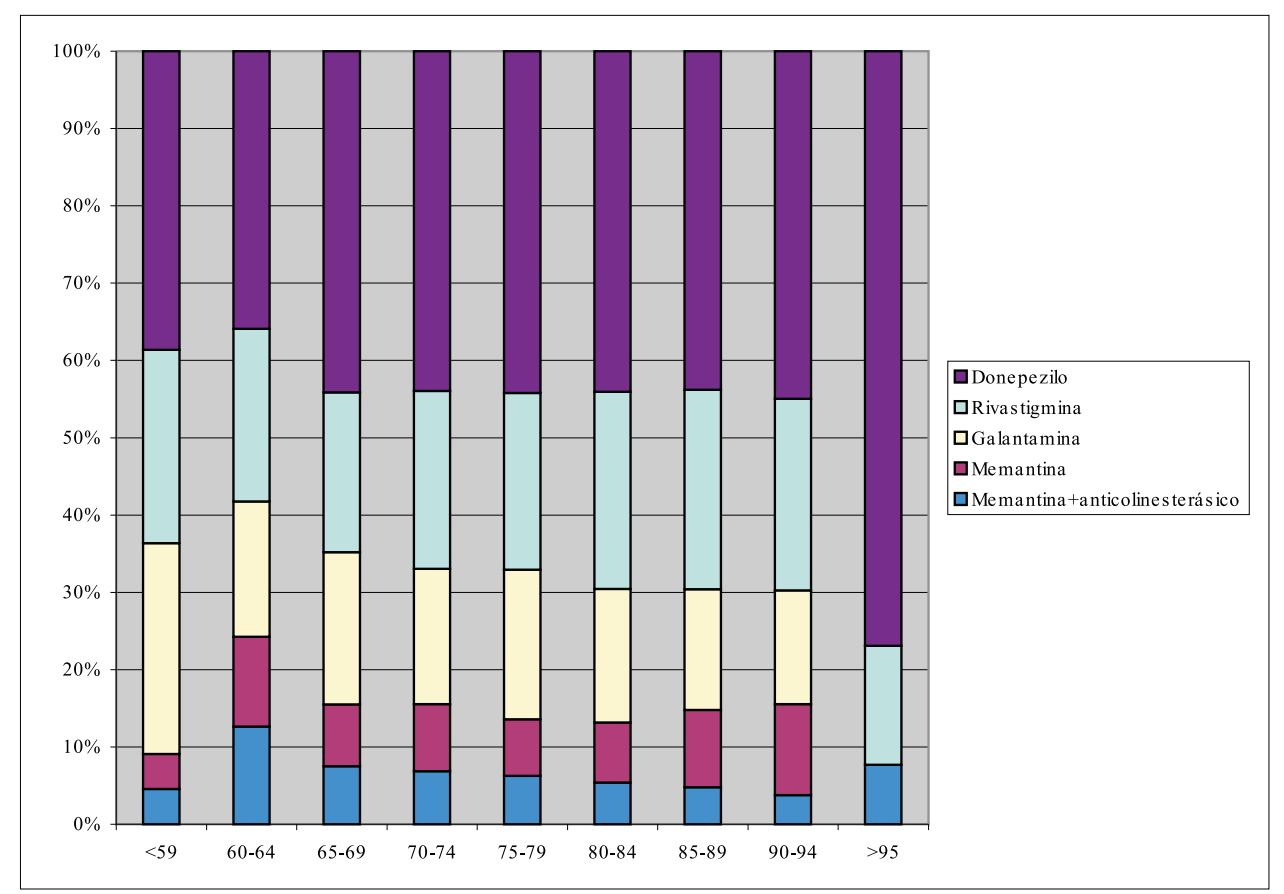

valencia por sexos indica que el sexo femenino es un factor asociado a presentar DTA a tratamiento farmacológico (OR: 1,68 [IC 95\%:1,58-1,79]).

En el figura 1 se muestra el porcentaje de pacientes en cada grupo de edad que esta siendo tratado con cada una de las opciones farmacoterapéuticas de la DTA. El medicamento más utilizado fue el donepezilo (44,46\% de los pacientes), no existiendo diferencias entre sexos. Por grupos de edad se apreciaron diferencias estadísticamente significativas en la utilización de donepezilo en los pacientes con edad igual o mayor a 95 años (75\% en 95 años vs. 46,9\%\% en < 95 años [ $p<0,03])$. También se hallaron diferencias estadísticamente significativas en la utilización de memantina (en monoterapia o combinada con alguno de los inhibidores de la acetilcolinesterasa) en los pacientes con edad igual o menor a 64 años $(19,7 \%$ en $=64$ años vs. $14 \%$ en $>64$ años [p<0,05]). Estas diferencias a favor de los pacientes $=64$ años en la utilización de memantina están causadas por el grupo de edad de 60 a 64 años, que es el que mayor porcentaje de utilización de memantina presenta $(24,27 \%$ en pacientes de 60 a 64 años vs. 13,95\% en el resto los grupos de edad $[\mathrm{p}<0,003])$.

El 5,49\% del total de pacientes tenían tratamientos combinados de memantina y un inhibidor de la acetilcolisterasa, correspondiendo el mayor porcentaje a la combinación de memantina con donepezilo $(2,93 \%$ del total de pacientes, frente a $1,58 \%$ de memantina con rivastigmina y un $0,98 \%$ de memantina con galantamina).

En el grupo de edad de $\geq 95$ años no se encontraron tratamientos con galantamina. 
Tabla 2

DDD/paciente/día de medicamentos antidemencia por principio activo

\begin{tabular}{|l|c|c|c|}
\hline Principio activo & DDD & DDD/paciente/día & IC95\% \\
\hline Donepezilo & $7,5 \mathrm{mg}$ & 1,18 & $1,17-1,20$ \\
\hline Rivastigmina & $9 \mathrm{mg}$ & 1,06 & $1,03-1,09$ \\
\hline Galantamina & $16 \mathrm{mg}$ & 1,17 & $1,15-1,20$ \\
\hline Memantina & $20 \mathrm{mg}$ & 0,99 & $0,98,1,00$ \\
\hline Memantina+Donepezilo & & 2,26 & $2,22-2,30$ \\
\hline Memantina+Rivastigmina & & 2,07 & $1,99-2,14$ \\
\hline Memantina+Galantamina & & 2,15 & $2,06-2,23$ \\
\hline
\end{tabular}

Tabla 3

DDD/paciente/día de medicamentos antidemencia por grupo de edad

\begin{tabular}{|c|c|c|c|c|}
\hline Grupos etáreos & Hombres & Mujeres & Total & DDD/paciente/día \\
\hline$\leq 59$ & 17 & 24 & 41 & 1,213 \\
\hline $60-64$ & 43 & 55 & 98 & 1,275 \\
\hline $65-69$ & 78 & 126 & 204 & 1,260 \\
\hline $70-74$ & 235 & 427 & 662 & 1,305 \\
\hline $75-79$ & 413 & 893 & 1306 & 1,264 \\
\hline $80-84$ & 446 & 1139 & 1584 & 1,249 \\
\hline $85-89$ & 231 & 725 & 956 & 1,203 \\
\hline $90-94$ & 43 & 190 & 233 & 1,187 \\
\hline \multirow[t]{2}{*}{$>95$} & 3 & 22 & 25 & 1,073 \\
\hline & 1.509 & 3.601 & & \\
\hline
\end{tabular}

En este grupo la memantina sólo se utilizó en terapia combinada con inhibidores de la acetilcolisterasa. En el resto de grupos de edad la memantina fue utilizada en monoterapia o combinada con alguno de los inhibidores de la acetilcolinesterasa. 
Figura 2

DDD/paciente/día de medicamentos antidemencia (por sexo y grupo de edad). Intervalos de confianza al $95 \%$ para la media

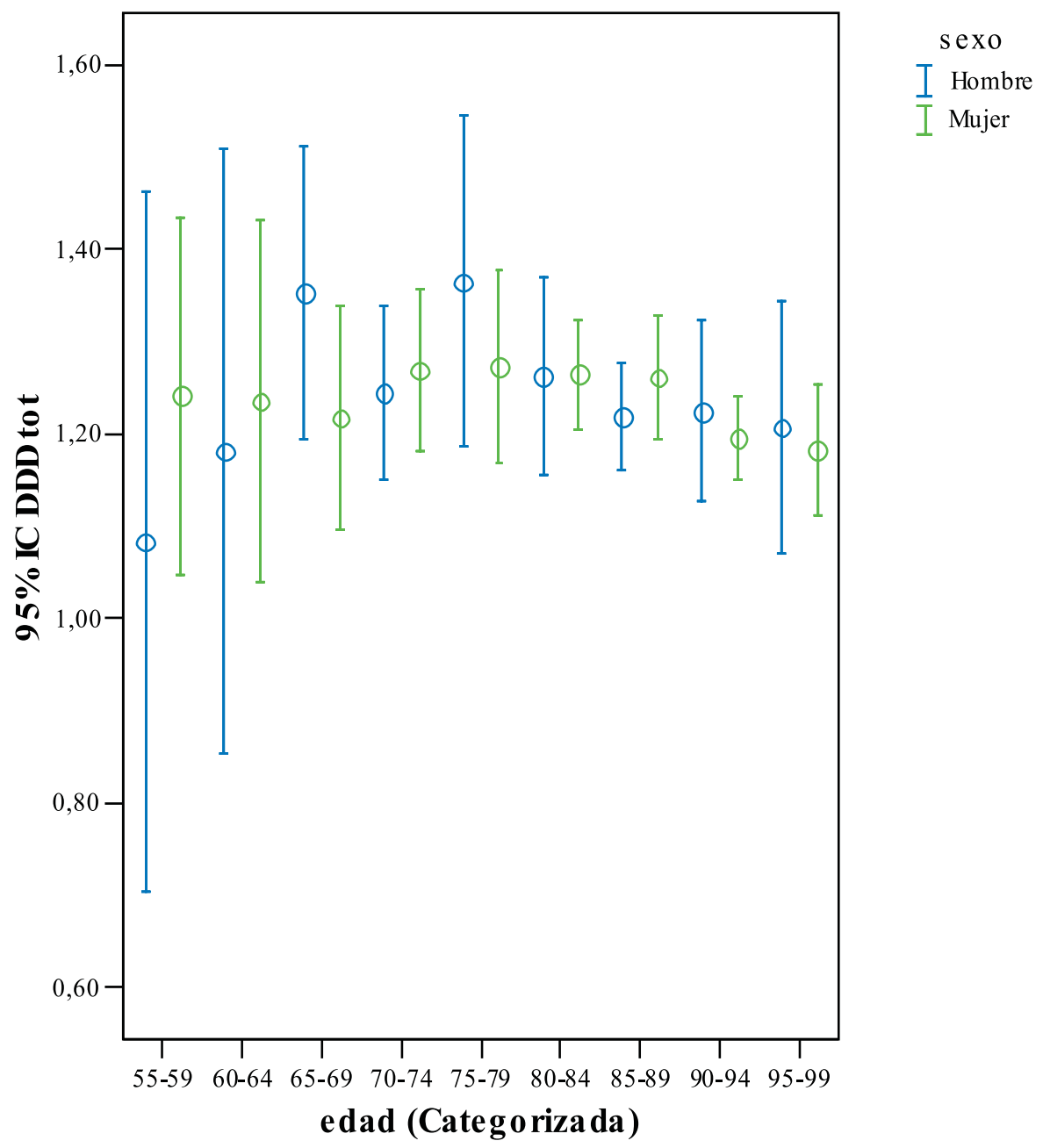

En la tabla 2 se muestran los datos de utilización de las distintas alternativas farmacoterapéuticas en el manejo de la DTA en la práctica clínica. Se expresan en DDD/ paciente/día, con intervalo de confianza del $95 \%$.

No se observaron diferencias estadísticamente significativas en la utilización de la terapéutica antidemencia, expresada en DDD/paciente/día, entre sexos, en concreto el consumo en varones ha sido de 1,25 DDD/paciente/día (IC95\% 1,21-1,30) y en mujeres 1,24 DDD/paciente/día IC95\% 1,21-1,27), ni entre grupos de edad (tabla 3), ni en la desagregación de los grupos de edad por sexo (Figura 2).

Rev Esp Salud Pública 2005, Vol. 79, N. ${ }^{\circ} 6$ 


\section{DISCUSIÓN}

En nuestro trabajo se observa que el principio activo más utilizado en la práctica clínica es el donepezilo. Aunque no existen estudios comparativos de eficacia entre los diferentes inhibidores de la acetilcolisterasa, el donepezilo es el más antiguo de los que se están utilizando actualmente en la práctica clínica (tacrina fue retirado por hepatotoxicidad en 1998). Existen estudios con diseño retrospectivo en los que donepezilo presentaba una menor tasa de abandonos que el resto de los inhibidores de la acetilcolistera$\mathrm{sa}^{13,14}$.

Hay que recordar que en el manejo de la DTA está formalmente contraindicada la utilización concomitante de dos inhibidores de la acetilcolinesterasa, por el aumento del riesgo de sus efectos adversos. En este estudio no se encontró ningún paciente que presentara este tipo de asociación.

En cuanto a la utilización de la memantina creemos que hay que diferenciar su utilización en monoterapia y la terapia combinada con inhibidores de la acetilcolinesterasa. Como ya se comentó previamente la memantina está autorizada en el tratamiento de la DTA moderada a grave. En nuestro estudio se hallaron diferencias estadísticamente significativas en el porcentaje de pacientes con memantina (con o sin combinación) a favor de los pacientes más jóvenes (=64 años). Este dato estaría justificado por ser este grupo de pacientes el que presenta una evolución más acelerada de la DTA.

Se ha formulado la hipótesis de que la asociación de fármacos con diferente mecanismo de acción para el tratamiento de la DTA podría resultar más eficaz que la administración de ambos principios activos por separado. En nuestro trabajo el 5,49\% de los pacientes presentan este tipo de asociación, correspondiendo el mayor porcentaje a la combinación de memantina con donepezilo (2,93\% del total de pacientes frente a $1,58 \%$ de memantina con rivastigmina y un $0,98 \%$ de memantina con galantamina). Aunque la evidencia es escasa, la combinación de memantina y donepezilo es la única que presenta evidencias científicas, en concreto un ensayo clínico aleatorizado doble ciego en el que a pacientes con donepezilo se les asociaba memantina o placebo ${ }^{15}$ y un estudio postcomercialización de seguridad en el que se estudiaba la misma asociación ${ }^{16}$. Los datos de estos estudios sugieren que esta combinación es segura y más eficaz que donepezilo solo, aunque no existen estudios que comparen memantina más donepezilo frente a memantina sola, que sería el comparador lógico al ser actualmente la memantina el techo terapeútico de la DTA.

No sabemos si este tipo de combinación se realiza en la práctica clínica de forma escalonada, es decir, si primero se inicia el tratamiento con el inhibidor de la acetilcolisterasa y después, tras la evolución de la enfermedad, se introduce la memantina, o si el patrón es desde el inicio la combinación de inhibidor de la acetilcolisterasa + memantina, aunque si se analiza la evolución de este tipo de tratamiento en los menores de 64 años, se observa un menor porcentaje de tratamientos combinados en el grupo más joven (menores de 59 años) que aumenta en el siguiente grupo de edad (60-64 años), estabilizándose en los siguientes grupos de edad y desapareciendo el uso en monoterapia de la memantina en los mayores de 95 años. Esto nos sugiere que la introducción tiende a realizarse en pacientes de peor pronóstico y de manera escalonada.

Los datos de nuestro trabajo en cuanto a la diferencia de prevalencias entre sexos son consistentes con estudios de prevalencia e incidencia de la demencia que muestran una tasa mayor en mujeres que en hombres, como ya se comentó en la introducción.

A pesar de que se desconoce la prevalencia real de la DTA en Galicia, en este estudio la prevalencia de pacientes con DTA con tra- 
tamiento farmacológico fue menor de lo esperado $^{17}$. Los datos hallados sugieren que la DTA es una patología infratratada en nuestra comunidad. Esto puede ser debido a que existen dudas sobre el beneficio terapéutico real de la medicación antialzheimer ${ }^{18}$ lo que puede inhibir a los médicos a su prescripción.

Dado que en nuestro trabajo se han detectado tratamientos combinados sobre los que no existe evidencia científica disponible (memantina con rivastigmina y memantina con galantamina) creemos necesario la realización de estudios que aporten evidencias sobre tolerabilidad y eficacia en esta clase de terapia combinada.

\section{BIBLIOGRAFÍA}

1. Warner J, Butler R. Enfermedad de Alzheimer. Evid Clin 2002;1:829-843.

2. Perkins P, Annegers JF, Doody RS, Cooke N, Aday L, Vernon SW. Incidence and prevalence of dementia in a multiethnic cohort of municipal retirees. Neurology 1997; 49:44-50.

3. Bachman DL, Wolf PA, Linn R, Knoefel JE, Cobb J. Prevalence of dementia and probable senile dementia of the Alzheimer type in the Framingham Study. Neurology 1992;42:115-119.

4. Fratiglioni L, Grut M, Forsell Y, Viitanen M, Grafstrom M. Prevalence of Alzheimer's disease and other dementias in an elderly urban population: relationship with age, sex, and education. Neurology 1991;41:1886-1892.

5. Folstein MF, Bassett SS, Anthony JC, Romanoski AJ, Nestadt GR. Dementia: case ascertainment in a community survey. J Gerontol 1991;46:132-138.

6. Jann M, Shirley K, Small G. Clinical pharmacokinetics and pharmaco-dynamics of cholinesterase inhibitors. Clin Pharmacokinet 2002;41:719-723.

7. Bonner L, Peskind E. Pharmacologic treatment of dementia. Med Clin North Am 2002; 86:657-74.
8. Centro Andaluz de Información de Medicamentos. Tratamiento de la enfermedad de Alzheimer ¿hay algo nuevo? Bol Ter Andal 2004; 20:17-20.

9. Committe for Proprietary Medicinal Products. European Public Assessment Report (EPAR). Axura®. European Agency for the Evaluation of Medicinal Products [en línea] URL disponible en <http://www.emea.eu.int/humandocs/Humans/EP AR/axura/axura.htm> . Citado el 10-5-2005].

10. Instituto Nacional de Estadística. Disponible en <http://www.ine.es Citado el 10-5-2005.

11. Servicio Gallego de salud. Base de datos SIAC-PF.

12. WHO Collaborating Centre for drug statistics methodology. Disponible en <http://www.whocc. no/atcddd/> Citado el 10-5-2005.

13. Broderick WC, Solis A, McRae T, Master V, Shah $\mathrm{S}$. Donepezil versus rivastigmine utilization patterns in a retrospective claims analysis. Baltimore, USA: AGS Annual Scientific Meeting; 2003.

14. Sicras A, Rejas-Gutiérrez J. Patrón de persistencia de tratamientos anticolinesterásicos en la demencia de tipo Alzheimer: análisis comparativo retrospectivo del donepezilo, la rivastigmina y la galantamina. Rev Neurol 2004;39: 312-16.

15. Tariot PN, Farlow MR, Grossberg GT, Graham SM, McDonald S, Gergel I; Memantine Study Group. Memantine treatment in patients with moderate to severe Alzheimer disease already receveing donepezil: a randomized controlled trial. JAMA 2004;291:317-24.

16. Hartmann S, Mobius HJ. Tolerability of memantine in combination with cholinesterase inhibitors in dementia therapy. Int Clin Psychopharmacol 2003 Mar;18:81-5.

17. Bermejo F, Trincado R. ¿Cuántas demencias y casos de enfermedad de Alzheimer hay en España? Actual Neurol Neurocienc Envejec 2003;1:341345 .

18. Kaduszkiewicz H, Zimmermann T, Beck-Bornholdt HP, van den Bussche H. Cholinesterase inhibitors for patients with Alzheimer's disease: systematic review of randomised clinical trials. BMJ. 2005 Aug 6;331(7512):321-7. 